\title{
p53-R273H promotes cancer cell migration via upregulation of neuraminidase- 1
}

Tao Lv ${ }^{1}$, Hong Lv², Junjie Fei ${ }^{3}$, Yajun Xie ${ }^{4}$, Daqing Lian ${ }^{1}$, Jiang Hu${ }^{1}$, Lizhou Tang ${ }^{1}$, Xiaodong Shi ${ }^{1}$, Jianling Wang 5 , Shibo Zhang ${ }^{2}$, Fengtian $\mathrm{Li}^{3}$, Xianjie Jiang ${ }^{6}$ and Yong $\mathrm{Yi}^{3}{ }^{\circledR}$

1. Center for Yunnan Plateau Biological Resources Protection Utilization, College of Biological Resource and Food Engineering, Qujing Normal University, Qujing, Yunnan, China 655011.

2. Hematology Department, The First People's Hospital of Qujing, Qujing, Yunnan, China 655000.

3. Center of Growth, Metabolism and Aging, and Key Laboratory of Bio-Resource and Eco-Environment, Ministry of Education, College of Life Sciences, Sichuan University, Chengdu, China 610064.

4. The Ministry of Education Key Laboratory of Laboratory Medical Diagnostics, the College of Laboratory Medicine, Chongqing Medical University, Chongqing, China 400016.

5. College of Chemistry and Environmental Science, Qujing Normal University, Qujing, Yunnan, China 655011.

6. The Key Laboratory of Carcinogenesis and Cancer Invasion of Ministry of Education, Cancer Research Institute, Central South University, Changsha 410078, China.

$\triangle$ Corresponding authors: E-mail: lvtao031@mail.qjnu.edu.cn (Tao Lv); Tel.: +86-874-889-8627; Fax: +86-874-889-8627; E-mail: yy-yiyong@scu.edu.cn (Yong Yi); Tel.: +86-28-8541-0034; Fax: +86-28-8541-0034.

(C) The author(s). This is an open access article distributed under the terms of the Creative Commons Attribution License (https://creativecommons.org/licenses/by/4.0/). See http:/ /ivyspring.com/terms for full terms and conditions.

Received: 2020.02.08; Accepted: 2020.09.20; Published: 2020.10.04

\begin{abstract}
Accumulating evidence indicates that hotspot p53 mutants have gain-of-function in promoting cell migration and tumor metastasis. However, the molecular mechanisms are not completely understood. Here, we show that a hotspot mutation, $\mathrm{p} 53-\mathrm{R} 273 \mathrm{H}$, promotes non-small cell lung cancer (NSCLC) cell migration and upregulates the mRNA and protein expression of neuraminidase-1 (NEUI), a sialidase involved in cell proliferation, cell migration and tumorigenesis. Silencing of NEU1 leads to upregulation of integrin $\beta 4$ which significantly inhibits NSCLC cell migration induced by $\mathrm{p} 53-\mathrm{R} 273 \mathrm{H}$. Mechanistically, p53-R273H promotes NEU1 transcription via activation of AKT signaling. Importantly, NEU1 expression is upregulated in human NSCLC samples harboring mutant p53 and is associated with poor clinical outcome. Overall, this study highlights an important role of NEU1 in p53-R273H-induced NSCLC cell migration and provides a potential target for NSCLC diagnosis and treatment.
\end{abstract}

Key words: p53-R273H; NEU1; ITGB4; AKT; cell migration

\section{Introduction}

Lung cancer is the leading cause of cancerrelated mortality and histologically is classified into small-cell lung cancer (SCLC) and non-small cell lung cancer (NSCLC). NSCLC accounts for approximately $80 \%-85 \%$ of all lung cancers [1, 2]. Mounting evidence indicates that proto-oncogenes, such as RAS, EGFR or PIK3CA, are often activated in NSCLC [3-5]. Moreover, the p53 tumor suppressor gene, the most studied gene in the human genome [6], is frequently mutated in NSCLC $[7,8]$. Accumulating clinical evidence indicates that mutant p53 is associated with NSCLC poor clinical outcome and cancer metastasis $[9,10]$.

The p53 protein contains five distinct domains, which include an N-terminal transactivation domain (TAD), a proline-rich domain (PRD), a DNA binding domain (DBD), an oligomerization domain (OD), and a C-terminal regulatory domain (CTD) [11]. Accumulating evidence indicates that $\mathrm{p} 53$ regulates a series of biological processes, including DNA damage repair, glucose metabolism, senescence, apoptosis and cell cycle arrest [12]. Several mutations clustered within the central DBD, referred to as "hotspot" mutations, are frequently found in cancers, refereed as "hotspot" mutations, and include R273, R175, G245, R248, R249 and R282 [13]. It has been documented that mutant p53 loses its original function and acquires gain of function (GOF) [14]. 
Mutant p53 can promote cancer cell survival, antiapoptosis, metastasis, angiogenesis, and resistance to chemotherapy [15-19]. At the molecular level, mutant p53 can directly bind to AMPK, the catalytic subunit of AMP-activated protein kinase (AMPK), to promote cancer cell proliferation and tumor growth [20]. Moreover, mutant $\mathrm{p} 53$ can promote integrin recycling or inhibit tight junction marker ZO-1 expression to facilitate cancer cell invasion and metastasis [18, 21]. We have previously shown that hotspot p53 mutant p53-R273H promotes the expression of Nrp2, resulting in cancer cell mobility and metastasis [17].

Neuraminidase-1 (NEU1) is a neuraminidase family protein that catalyzes the removal of sialic acid residues from the glycan chains of glycoproteins, oligosaccharides and sialylated glycolipids and modulates molecular and cellular recognition events $[22,23]$. It has been reported that NEU1 plays a critical role in sialidase-mediated tumorigenesis [24]. Recently, it has been documented that NEU1 has a potential role in the regulation of cell proliferation, migration, invasion and cancer metastasis [25-27].

In this study, we show that mutant p53 (p53$\mathrm{R} 273 \mathrm{H})$ promoted NEU1 transcription via activation of AKT which leads to downregulation of ITGB4, consequently resulting in increased NSCLC cell mobility. Silencing of NEU1 or AKT inhibitor treatment significantly inhibited p53-R273H-induced NSCLC cell migration. Together, this study highlights the role of NEU1 in mutant p53-induced NSCLC cell migration and provides a potential target for NSCLC diagnosis and treatment.

\section{Materials and Methods}

\section{Cell culture and drug treatment}

Human non-small cell lung cancer (NSCLC) H1299 and HEK 293T (human embryonic kidney) cells were cultured in DMEM (Gibco, Rockville, MD, USA) supplemented with $10 \%$ fetal bovine serum (FBS; HyClone, Logan, UT, USA). Human NSCLC H1975 cells were cultured in RPMI-1640 medium (Gibco) supplemented with 10\% FBS (HyClone). All cells were grown in medium supplemented with 100 units/mL penicillin (Gibco) and $100 \mu \mathrm{g} / \mathrm{mL}$ streptomycin (Gibco). Cells were maintained in a humidified $37{ }^{\circ} \mathrm{C}$ incubator under a $5 \% \quad \mathrm{CO}_{2}$ atmosphere. Cells at $75-85 \%$ confluence were treated with the indicated chemical compound. MK2206 (S1078) was purchased from Selleck Chemicals (Houston, USA).

\section{Plasmid transfection and lentiviral infection}

Cells at $70-80 \%$ confluence were transfected using Lipofectamine 2000 transfection reagent
(Invitrogen). Recombinant lentiviruses were amplified by co-transfecting HEK 293T cells with the psPAX2 and pMD2.G packaging plasmids and p53-R273H, NEU1 or ITGB4 lentiviral expression plasmid using Lipofectamine 2000. Viruses were collected at 60 hours after transfection. Cells at 50\% confluence were infected with a recombinant lentivirus in the presence of $10 \mu \mathrm{g} / \mathrm{mL}$ polybrene, followed by 12 hours of incubation at $37^{\circ} \mathrm{C}$ with $5 \%$ $\mathrm{CO}_{2}$. Lentiviral-based shRNAs specific for green fluorescent protein (GFP) (GAAGCAGCACGACTTC TTC), p53 (\#1 AAG-ACTCCAGTGGTAATC TACT; \#2 CACCATCCACTACAACTACAT), NEU1 (\#1 CCCGGAATCT CTCCCTGGATA; \#2 GCTTCAGC AATGGTACCTCAT) and ITGB4 (AAGAACCGGAT GCTGCTTATT) were constructed as described [28].

\section{Western blot analyses}

Cells were lysed in $\mathrm{EBC}_{250}$ lysis buffer $(250 \mathrm{mM}$ $\mathrm{NaCl}, 25 \mathrm{mM}$ Tris, $\mathrm{pH}$ 7.4, 0.5\% Nonidet P-40, $50 \mathrm{mM}$ $\mathrm{NaF}$, and supplemented with $0.5 \mathrm{mM} \mathrm{Na}_{3} \mathrm{VO}_{4}, 0.2$ $\mathrm{mM}$ phenylmethylsulfonyl fluoride, $20 \mu \mathrm{g} / \mathrm{mL}$ aprotinin and $10 \mu \mathrm{g} / \mathrm{mL}$ leupeptin). Equal amounts of total protein were separated by SDS-PAGE, transferred to PVDF membrane and hybridized to an appropriate primary antibody and HRP-conjugated secondary antibody for subsequent detection by enhanced chemiluminescence. Antibodies to detect the following proteins were used in western blotting: p53 DO-1 (1:1000, Santa Cruz Biotechnology), NEU1 (1:1000, Abcam), Akt (1:1000, Abcam), pAkt (Ser473, 1:2000, Abcam), Integrin $\beta 4$ (1:1000, 4707, Cell Signaling Technology), and GAPDH (1:2000, Cell Signaling Technology).

\section{Quantitative PCR}

For quantitative PCR (QPCR) analysis, total RNA was isolated from cells using an RNA extraction kit (Qiagen, Germany) according to the manufacturer. Complementary DNA (cDNA) was generated using the first-strand cDNA kit (TAKARA, Inc). QPCR analyses were performed in a CFX96 Real-Time PCR System (Bio-Rad) using SoFast EvaGreen Supermix (Bio-Rad), according to the manufacturer's instructions. The reactions were carried out in a 96-well plate at $95^{\circ} \mathrm{C}$ for $2 \mathrm{~min}$, followed by 35 cycles of $95^{\circ} \mathrm{C}$ for $15 \mathrm{~s}$ and $55^{\circ} \mathrm{C}$ for $30 \mathrm{~s}$. GAPDH was used as a reference. QPCR primers specific for GAPDH (F: TGGACTCCACGACGTACTCA; R: AATCCCATCA CCATCTTCCA); NEU1 (F: TGTGACCTTCGACCC TGAGC; R: TCGCAGGGTCAGGTTCACTC); DDR1 (F:5'-CATGAGCCGGAACCTCTA-3'; R: 5'-CCACAG GGTCACACCAAA-3'); KRT8 (F:5'-CTGTCCATGAA GGATGACTT-3'; R: 5'-TGTCCACTCTGTCTGTG AGA-3'); GRAMD1B (F: 5'-TGGGGGAGAAGATTGA 
GATG-3' ; R: 5'-TGTCC A CGCTGAAGTTGAAG-3'); LTBP3 (F: 5'-CGGAACGGAGTGTGTGAGAA- 3'; R:5'-CTCGTCCACGTCCATCTCT-3'); TNFRSF12A (F: 5'-CTGGCTCC AGAACAGAAAGG-3'; R: 5'-GGG CCTAGTGTCAAGTCTGC-3'); and ITGB4 (F: 5'-CAC CGCGTGCTAAGCACAT-3'; R: 5'-TGTGGTCGAGT GAGTGTTCTG-3') were used.

\section{Transwell assays for cell migration}

Transwell assays were performed as described [29]. Briefly, cell migration was measured using $6.5 \mathrm{~mm}, \quad 8.0 \mu \mathrm{m}$-pore polycarbonate membrane transwell inserts (BD Biosciences, San Jose, CA, USA). Cells were suspended in serum-free media and seeded into the inner chamber $\left(\mathrm{H} 1299,0.5 \times 10^{5}\right.$ cells per chamber; H1975, $2.0 \times 10^{5}$ cells per chamber). The outer chamber contained complete growth media. Cells were incubated for 24 hours. Nonmigrating cells were carefully removed with a cotton swab. Migrating cells were stained with $0.4 \%$ crystal violet in methanol for $20 \mathrm{~min}$ at room temperature and imaged under a Zeiss light microscope.

\section{Bioinformatics analysis}

The Oncomine and TCGA databases were used to analyze NEU1 mRNA levels in clinical human lung cancers. The Kaplan-Meier plotter platform (https:// kmplot.com/analysis/) was used to analyze the overall survival of lung cancer patients.

\section{Statistical analysis}

GraphPad Prism 6.0 (GraphPad Software Inc. USA) was used for data recording, collection, processing, and calculation. ImageJ (Rawak Software, Inc. Germany) software was used to analyze cell migration. All experiments were performed at least three times in triplicate. Quantitative data were analyzed statistically using Student's $t$-test to assess significance.

\section{Results}

\section{p53-R273H promotes NEU1 expression}

Accumulating evidence indicates that mutant p53, such as $\mathrm{R} 273 \mathrm{H}$ or $\mathrm{R} 175 \mathrm{H}$, plays a critical role in NSCLC cell migration and cancer metastasis [18, 21]. To investigate the underlying mechanism by which mutant p53 promotes NSCLC cell migration, we first performed RNA-Seq analyses. As shown in Figure $1 \mathrm{~A}$, overexpression of p53- $\mathrm{R} 273 \mathrm{H}$ significantly upregulated the expression of a series of cell migrationrelated genes, including NEU1, KERT8, DDR1, LTBP3, and GRAMD1B, in NSCLC H1299 cells that lack endogenous p53. Furthermore, QPCR analyses
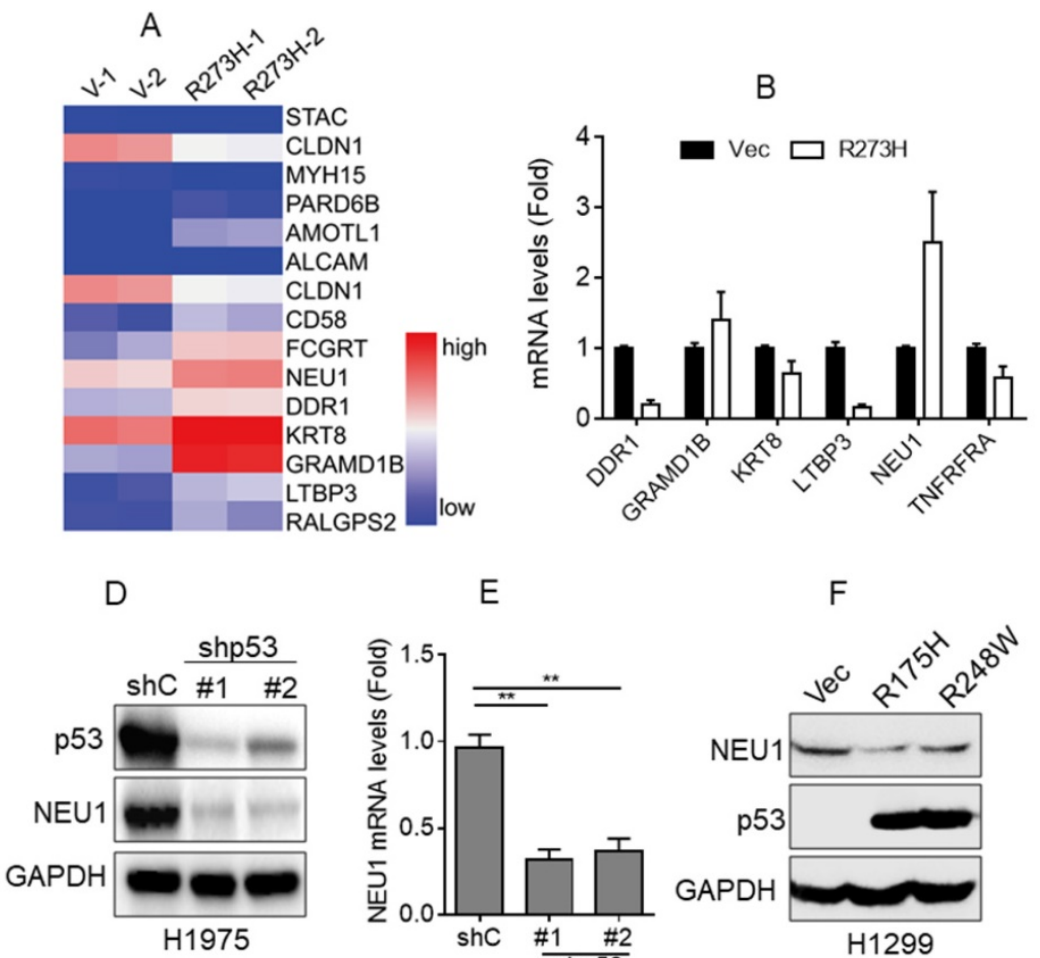

E

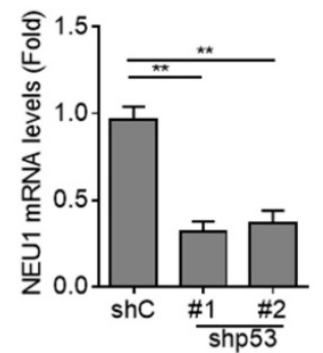

$\mathrm{F}$

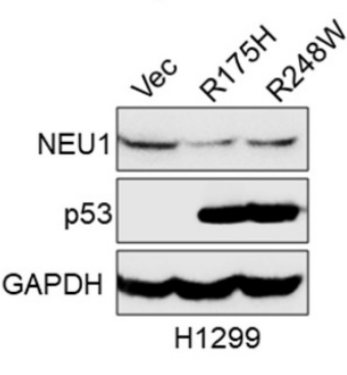

C

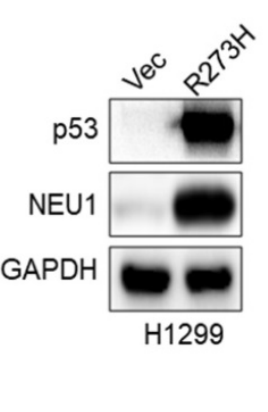

G

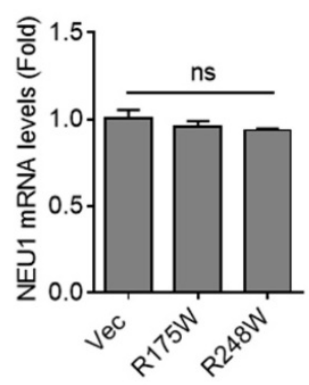

Figure 1. p53-R273H promotes NEU1 expression. (A) H1299 cells stably expressing vector control (Vec) or mutant p53-R273H (R273H) were subjected to RNA-seq analyses in duplicate. A heatmap showing changes of differentially expressed genes related to cell migration. (B-C) $\mathrm{H} 1299$ cells stably expressing Vec or R273H were subjected to Q-PCR (B) or Western blot (C) analyses.(D-E) H1 975 cells stably expressing shGFP (shC) or shRNA specific for p53 (shp53-1, shp53-2) were subjected to Western blot (D) or Q-PCR analyses (E). (F-G) H1299 cells stably expressing Vec, p53-R175H or p53-R238W were subjected to Western blot (F) or Q-PCR (G) analyses. Data are presented as means \pm SEM. $* *, p<0.01$. 
showed that overexpression of p53-R273H notably increased NEU1 mRNA levels, consistent with the RNA-Seq data (Figure 2B). To further examine the effects of p53-R273H on NEU1 protein expression, we performed Western blot analyses in $\mathrm{H} 1299$ cells. As shown in Figure 1C, overexpression of p53-R273H also significantly increased NEU1 protein expression.
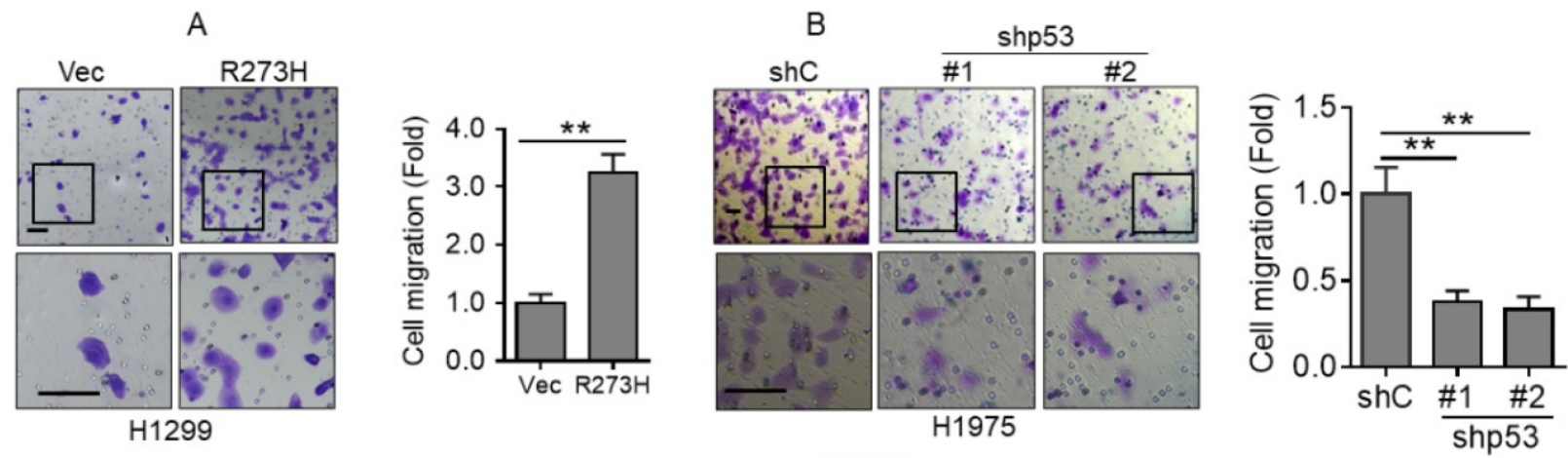

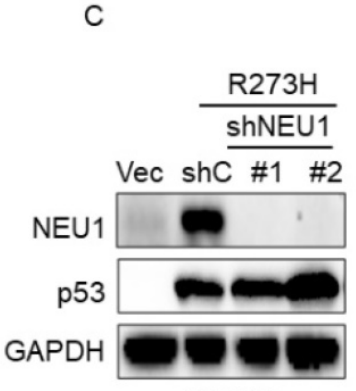

H1299

E

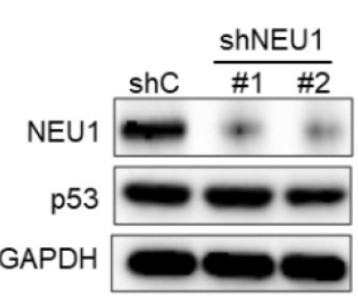

H1975

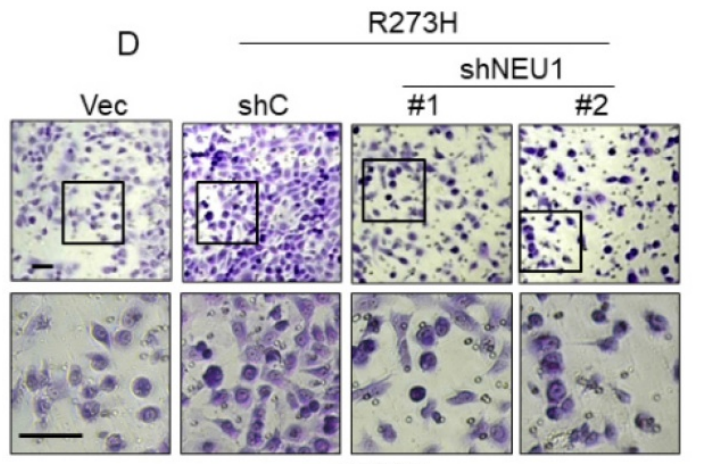

$\mathrm{H} 1299$

$\mathrm{F}$

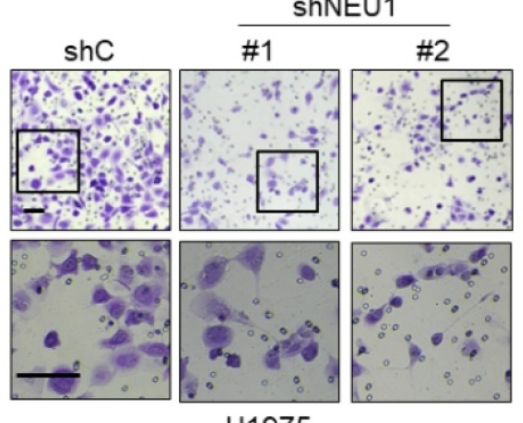

$\mathrm{H} 1975$
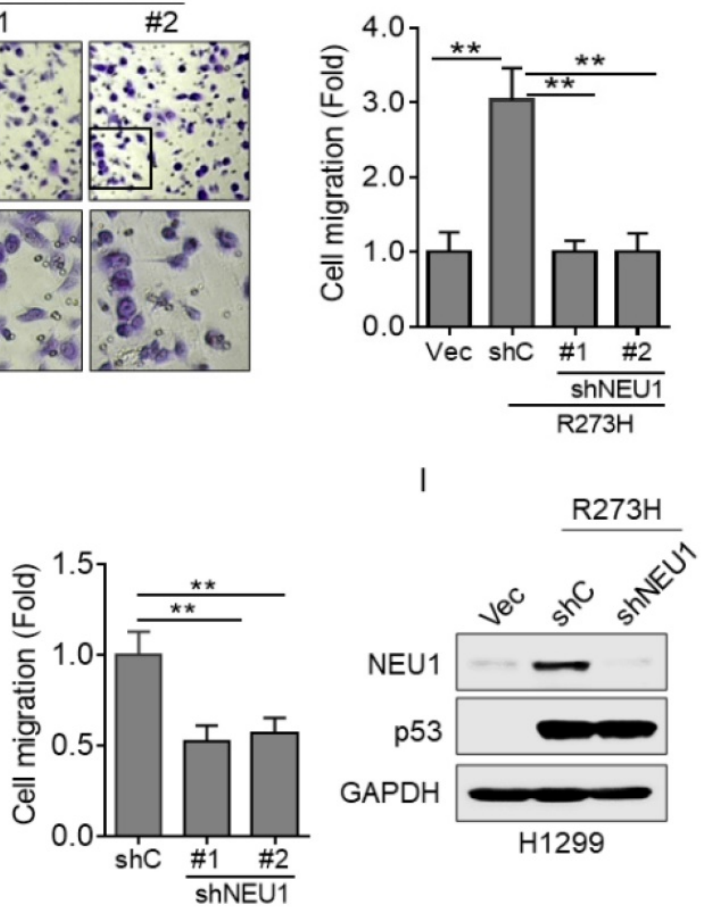

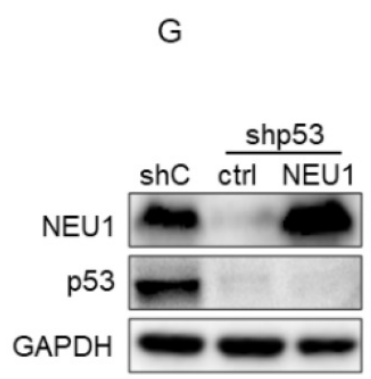

H1975

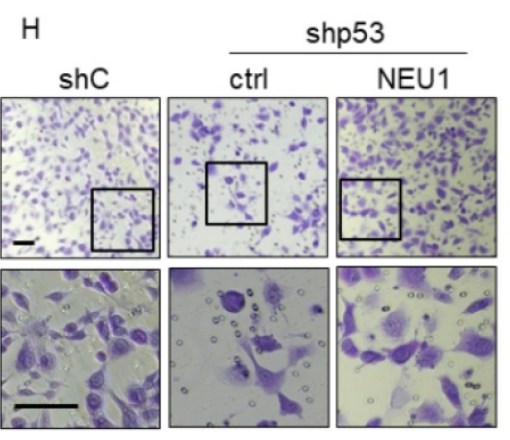

H1975

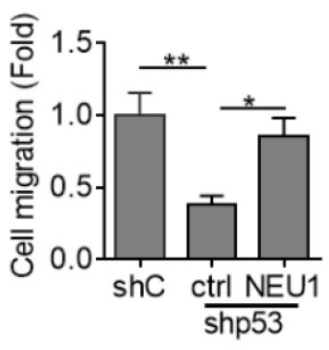

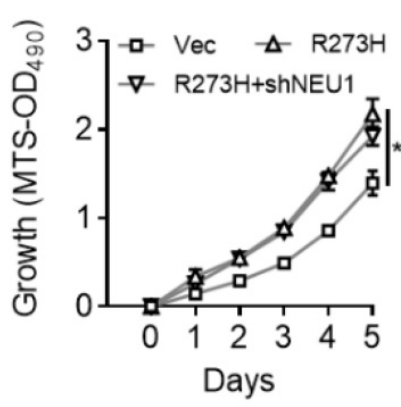

Figure 2. p53-R273H promotes cell migration via upregulation of NEU1. (A) $\mathrm{H} 1299$ cells stably expressing Vec or $\mathrm{R} 273 \mathrm{H}$ ( $\mathrm{H} 1299-\mathrm{R} 273 \mathrm{H}$ ) were subjected to transwell assay for cell migration. (B) H1975 cells stably expressing shC, shp53-1 or shp53-2 were subjected to transwell assay for cell migration. (C-D) H1299-R273H cells stably expressing a control shRNA (shC) or shRNA specific for NEU1 (shNEU1-1 or shNEU1-2) were subjected to Western blot analyses(C) or to transwell assay for cell migration(D). (E-F) H1975 cells stably expressing shC, shpNEU1-1 or shpNEU1-2 were subjected to Western blot analyses or to transwell assay for cell migration. (G-H) H1975-shp53 cells stably expressing NEU1 were subjected to Western blot $(\mathrm{G})$ or to transwell assay for cell migration $(\mathrm{H})$. (I-J) $\mathrm{H} 1299-\mathrm{R} 273 \mathrm{H}$ cells stably expressing shC or shNEU1 were subjected to Western blot analyses (I) or to MTS assay for cell growth ability (J). Data are presented as means \pm SEM. Scale bar $=100 \mu \mathrm{m}$. **, $p<0.01 ; *, p<0.05$. 
It has been reported that NSCLC H1975 cells harbor an endogenous p53-R273H mutation [30]. Therefore, we wondered whether silencing endogenous p53-R273H could decrease NEU1 expression. To confirm this hypothesis, we stably expressed shRNA specific for p53 in H1975 cells. As shown in Figure $1 \mathrm{D}$ and 1E, knockdown of endogenous p53-R273H dramatically decreased NEU1 mRNA and protein levels.

In addition, we examined the effects of the other mutant p53, such as R175H and R248W, on NEU1 expression. As shown in Figure $1 \mathrm{~F}$ and $1 \mathrm{G}$, ectopic expression of p53-R175H or p53-R248W had little effects on NEU1 protein and mRNA expression in H1299 cells.

Together, these results indicate that p53-R273H can effectively promote NEU1 expression.

\section{NEU1 plays a pivotal role in p53-R273H- induced cell migration}

To investigate the role of NEU1 in p53-R273Hinduced NSCLC cell migration, we first examined the effects of p53-R273H on cell migration in our system. As shown in Figure 2A, overexpression of p53-R273H significantly increased H1299 cell migration, as evidenced by transwell analyses. Conversely, silencing endogenous p53-R273H in H1975 cells dramatically inhibited cell migration (Figure 2B). Next, to examine the role of NEU1 in p53-R273Hinduced cell migration, we silenced NEU1 in H1299$\mathrm{R} 273 \mathrm{H}$ cells. As shown in Figure $2 \mathrm{C}$ and 2D, knockdown of NEU1 significantly inhibited H1299 cell migration induced by p53-R273H. Moreover, silencing endogenous NEU1 in H1975 cells also significantly inhibited cell migration (Figure 2E and 2F). Importantly, knockdown of endogenous p53-R273H in H1975 cells dramatically inhibited cell migration, which was significantly rescued by overexpression of NEU1 (Figure 2G and 2H). It has been documented that $\mathrm{p} 53-\mathrm{R} 273 \mathrm{H}$ can promote cell proliferation and tumor growth [31]. We therefore examined the effects of NEU1 on p53-R273H-induced cell proliferation. As shown in Figure 2I and 2J, consistent with previous report, p53- $2273 \mathrm{H}$ can significantly increase H1299 cell proliferation; however, silencing of NEU1 had little effects on p53-R273H-induced H1299 cell proliferation. Together, these results demonstrate that NEU1 plays a critical role in p53-R273H-mediated upregulation of NSCLC cell migration.

\section{Integrin $\beta 4$ is critical in p53-R273H-NEU 1 axis-induced cell migration}

Integrin $\beta 4$ (ITGB4), an important cell-matrix adhesion molecule, plays a pivotal role in cell migration [32]. It has been reported that NEU1 is critical in regulating ITGB4-mediated signaling [33]. Therefore, we speculated that ITGB4 may play a role in the p53-R273H/NEU1 axis-induced NSCLC cell migration. To investigate this hypothesis, we first examined the effects of NEU1 on ITGB4 expression. As shown in Figure 3A and 3B, silencing of NEU1 significantly increased ITGB4 protein and mRNA expression. Moreover, overexpression of p53-R273H significantly increased NEU1 expression concomitant with decreased ITGB4 expression (Figure 3C). Silencing of NEU1 dramatically rescued p53-R273Hinduced downregulation of ITGB4 expression (Figure 3C). Furthermore, overexpression of ITGB4 significantly inhibited p53-R273H-induced upregulation of cell migration (Figure 3D). Consistently, knockdown of NEU1 in H1975 cells significantly increased ITGB4 expression as well as inhibited cell migration (Figure 3F and 3G). Importantly, silencing of NEU1-induced downregulation of cell migration was dramatically rescued by knockdown of ITGB4 (Figure $3 \mathrm{~F}$ and $3 \mathrm{G}$ ). Together, these results indicate that ITGB4 plays a role in p53-R273H-NEU1 axis-induced cell migration.

\section{p53-R273H promotes NEU1 expression via activation of AKT signaling}

Next, we examined the underlying mechanism by which p53-R273H promotes NEU1 expression in NSCLC cells. It has been documented that mutant p53 can activate AKT signaling by promoting epidermal growth factor receptor (EGFR) trafficking [18]. Therefore, we speculated that AKT signaling may be involved in p53-R273H-mediated upregulation of NEU1 expression. To confirm this hypothesis, we used MK2206, an AKT specific inhibitor, to treat H1299-R273H cells. As shown in Figure 4A and 4B, inhibition of AKT activity significantly suppressed p53-R273H-induced NEU1 mRNA and protein expression. Moreover, p53-R273H-induced downregulation of ITGB4 protein expression also significantly was rescued by AKT inhibition. Furthermore, p53-R273H-induced H1299 cell migration was also dramatically inhibited by MK2206 treatment (Figure 4C). In addition, inhibition of AKT activity in H1975 cells also significantly inhibited NEU1 protein and mRNA levels concomitant with increased ITGB1 protein expression and decreased cell migration (Figure 4D-4F). Overexpression of NEU1 significantly rescued inhibition of AKTinduced upregulation of ITGB1 protein expression and downregulation of cell migration (Figure 4D-4F). Together, these results indicate that p53-R273H promotes NEU1 expression and cell migration through activation of AKT signaling. 

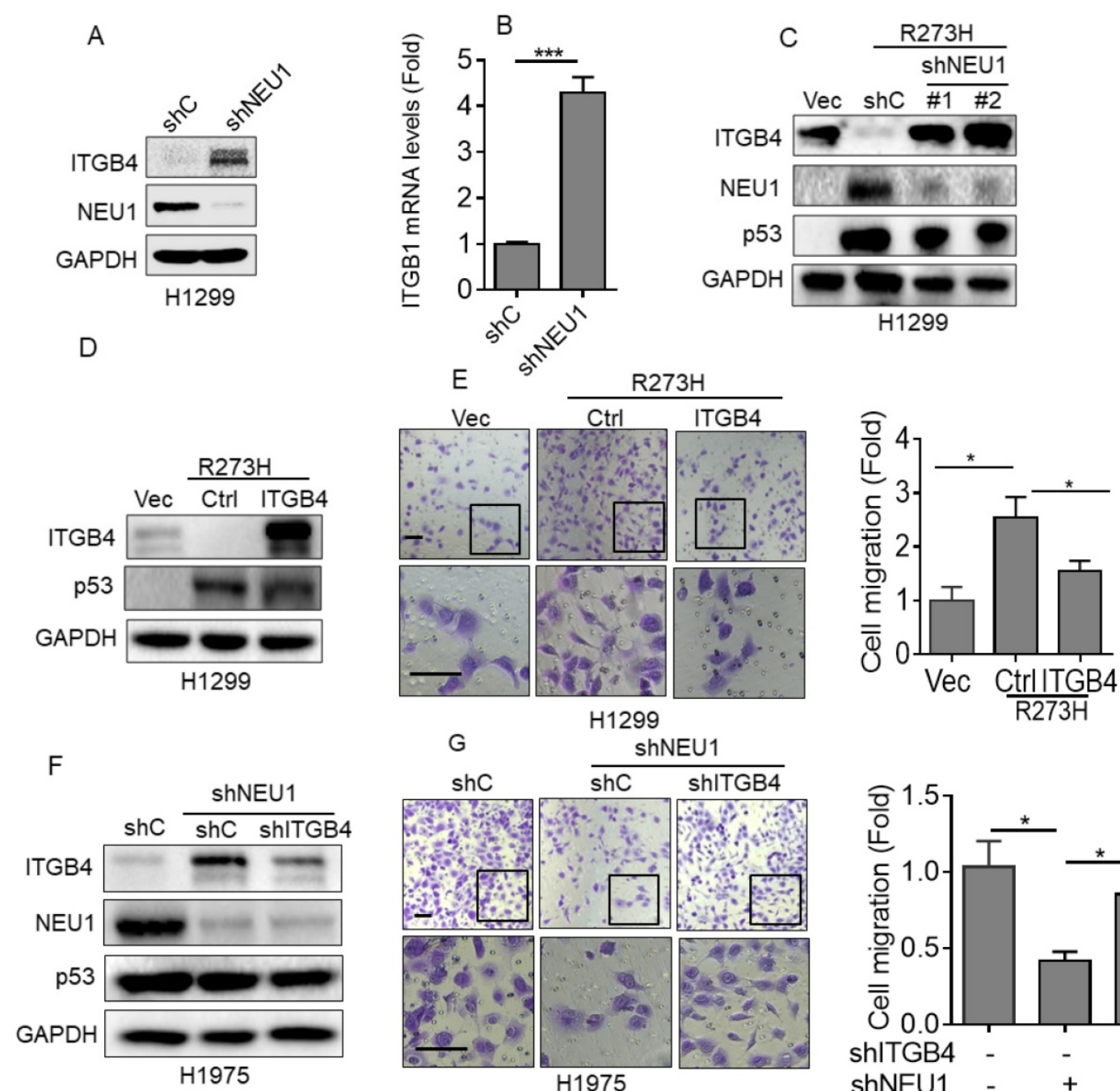

Figure 3. Integrin $\beta 4$ is critical in p53-R273H-NEU1 axis-induced cell migration. (A-B) $\mathrm{H} 1299$ cells stably expressing shC or shNEU1 were subjected to Western blot (A) or Q-PCR (B) analyses. (C) H1299-R273H cells stably expressing shC, shNEU1-1 or shNEU1-2 were subjected to Western blot analyses. (D-E) H1299-R273H cells stably expressing integrin $\beta 4$ (ITGB4) or a vector control (Ctrl) were subjected to Western blot analyses (D) or to transwell assay for cell migration (E). (F-G) H1975-shNEU1 cells stably expressing shC or shITGB1 were subjected to Western blot analyses (F) or to transwell assay for cell migration (G). Data are presented as means \pm SEM. Scale bar $=100$ $\mu \mathrm{m} . *, p<0.05$.

\section{NEU1 is upregulated in human NSCLC harboring mutant p53 and is associated with poor clinical outcome}

To determine the clinical relevance of NEU1 expression in human NSCLC, we first used the Oncomine database to analyze NEU1 mRNA levels in human NSCLC carcinoma tissues. As shown in Figure $5 \mathrm{~A}$ and $5 \mathrm{~B}$, compared to normal lung tissues, NEU1 mRNA levels were significantly increased in NSCLC carcinoma tissues. Moreover, high NEU1 mRNA levels in NSCLC patients correlated with a short overall survival (Figure 5C). Importantly, compared to wild-type p53 NSCLC carcinoma tissues, mutant p53 NSCLC carcinoma tissues exhibited higher NEU1 mRNA expression (Figure 5D). Together, these clinical results indicate that NEU1 mRNA expression is correlated with p53 status and is associated with NSCLC patients' clinical outcome.

\section{Discussion}

Accumulating clinical evidence indicates that p53 is the most commonly mutated gene in human cancer [34]. It has been reported that mutant p53 is associated with poor clinical outcome in NSCLC patients [35]. Mutant p53 regulates multiple biological functions, including cell proliferation, tumor growth and cancer metastasis [14, 17]. It has been documented that mutant p53 can promote integrin recycling or inhibit ZO-1 expression to enhance cell invasion and cancer metastasis $[18,21]$. Our previous study demonstrated that p53-R273H significantly promotes cell migration and cancer metastasis via upregulation of Nrp2 expression [17]. In this study, we showed that NEU1 mRNA expression is associated with NSCLC patient overall survival. NSCLC patients with high NEU1 mRNA expression exhibited low overall survival. Importantly, we found 
that NEU1 is a novel downstream target of p53$\mathrm{R} 273 \mathrm{H}$ and plays an important role in p53-R273Hinduced NSCLC cell migration (Figure 5E). However, we found that the others p53 hotspot mutations, such as $\mathrm{R} 175 \mathrm{H}$ or $\mathrm{R} 248 \mathrm{~W}$, have little effects on NEU1 expression, suggesting that $\mathrm{p} 53-\mathrm{R} 273 \mathrm{H}$ may specific regulate NEU1 expression.

The question remains how p53- $\mathrm{R} 273 \mathrm{H}$ promotes NEU1 expression. It has been demonstrated that activated AKT signaling regulates a series of gene transcription pathways, including c-Myc and p63 [36]. Interestingly, it has been shown that mutant p53 can activate AKT signaling by enhancing EGFR trafficking [18]. We showed that p53-R273H significantly promotes NEU1 transcription. Therefore, we speculate that AKT signaling may play a role in p53-R273H promoting NEU1 expression. Indeed, inhibition of AKT dramatically suppressed p53$\mathrm{R} 273 \mathrm{H}$-induced NEU1 mRNA and protein expression contaminant with reduced cell migration. Our previous data indicate that AKT activation can directly phosphorylate FOXO3a, which leads to the inhibition of p63 transcription [29]. However, whether FOXO3a is also involved in AKT-mediated regulation of NEU1 transcription needs to be further examined. Moreover, it is reported that $\mathrm{p} 53-\mathrm{R} 273 \mathrm{H}$ can be used as a transcriptional co-factor binds to G/C-rich regulatory regions to active gene expression [37]. There are serval G/C-rich regions on NEU1 gene promoter. Therefore, p53-R273H may regulate NEU1 transcription through both direct and indirect manners. However, whether p53-R273H direct binds to NEU1 gene promoter needs to further investigate.

NEU1 functions as a sialidase to regulate a set of genes involved in a variety of different biological processes, including cell migration and cancer metastasis [38, 39]. Integrin family proteins, as cell surface adherent receptors, are fundamentally important for multiple facets of biological processes, including cell-cell junctions, metastasis and survival $[18,32,40]$. Accumulating evidence indicates that integrin $\beta 4$, an important integrin family protein, suppresses cancer metastasis via inhibition of EMT and promotion of cell-cell and cell-matrix adhesion [32]. Interestingly, it has been reported that NEU1 plays a critical role in the regulating ITGB4-mediated signaling [33]. Given these observations, ITGB4 may play a role in the p53-R273H/NEU1 axis-induced NSCLC metastasis. Indeed, our results show that ITGB4 plays a pivotal role in NEU1-induced NSCLC cell migration.

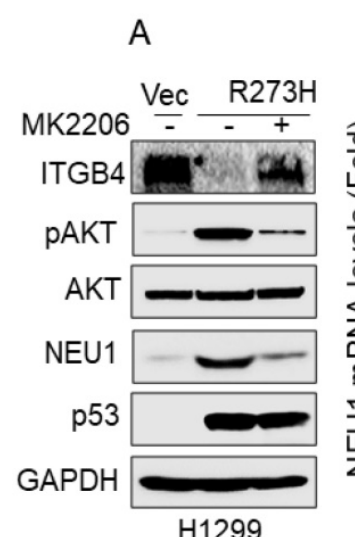

$\mathrm{H} 1299$

$\mathrm{D}$

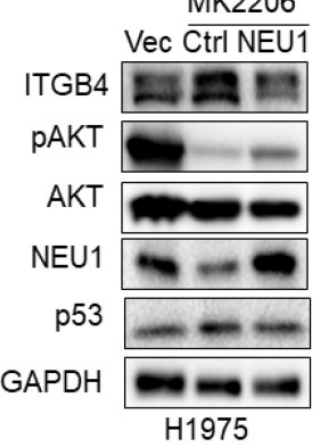

B

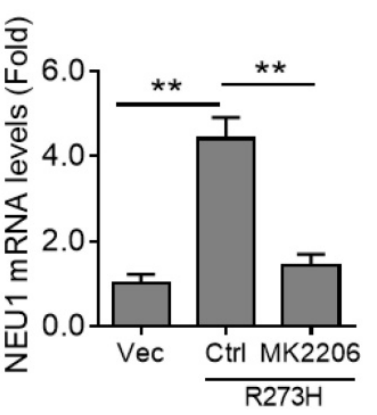

$\mathrm{E}$

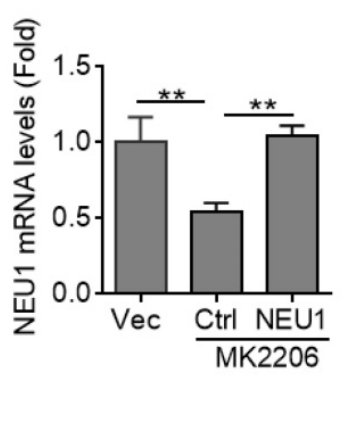

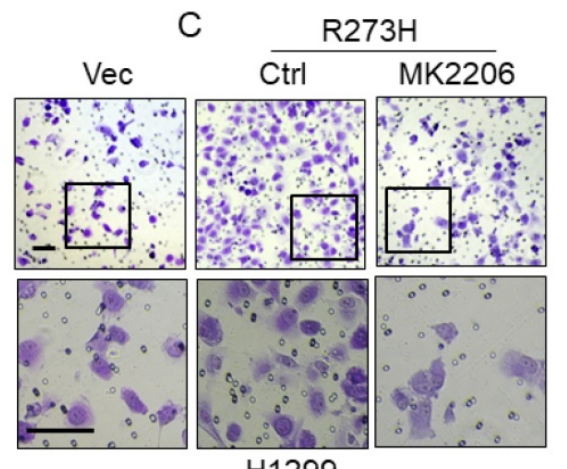

$\mathrm{H} 1299$

F

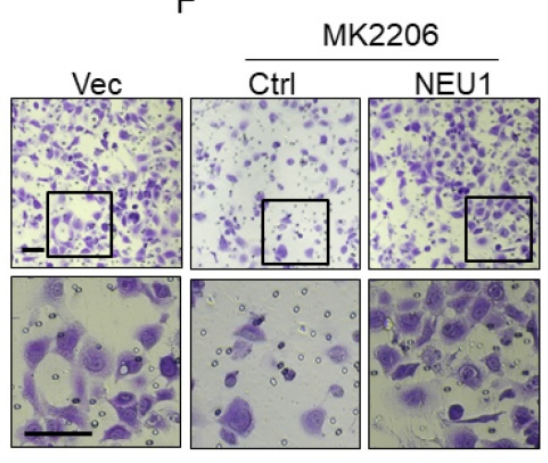

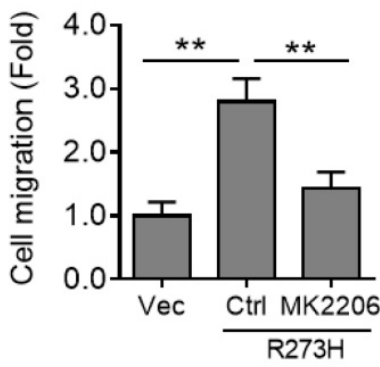

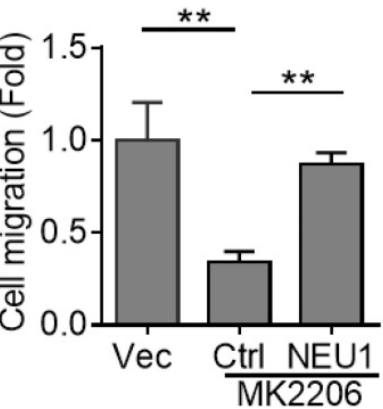

Figure 4. p53-R273H promotes NEU1 transcription via activating AKT. (A-C) H1299-R273H cells were treated or untreated with MK2206 (10 $\mu$ M) for 24 h. Cells were subjected to Western blot (A) or Q-PCR (B) analyses or were subjected to transwell assay for cell migration (C). (D-F) H1975 cells were treated or untreated with MK2206 $(10 \mu \mathrm{M})$ for $24 \mathrm{~h}$. Cells were subjected to Western blot $(\mathrm{D})$ or Q-PCR $(\mathrm{E})$ analyses or were subjected to transwell assay for cell migration $(\mathrm{F})$. Data are presented as means \pm SEM. Scale bar $=100 \mu \mathrm{m}$. ${ }^{* *}, p<0.01$. 

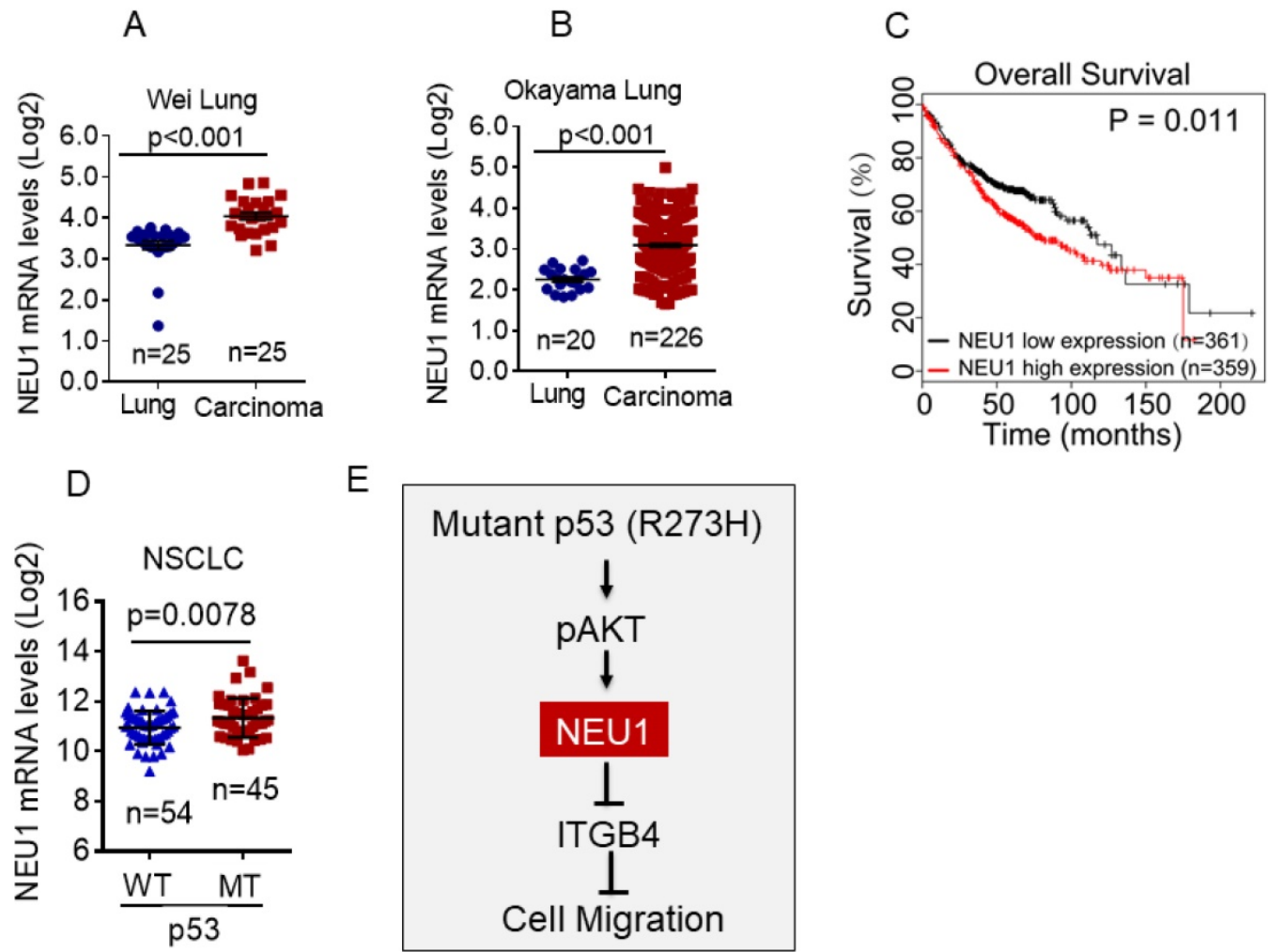

Figure 5. NEU1 is upregulated in human NSCLC harboring mutant p53 and is associated with poor clinical outcome. (A-B) The Oncomine Wei_Lung cancer dataset or Okayama lung cancer dataset was analyzed for NEU1 mRNA levels in human normal lung or lung carcinoma. (C) The overall survival in lung cancer patients were analyzed using Kaplan-Meier database. (D) The TCGA database was analyzed for NEU1 mRNA levels in human male NSCLC bearing wild type p53 alleles ( $\mathrm{p} 53$-WT) or $\mathrm{p} 53$ mutant alleles (p53-MT). (E) A model depicts the role of NEU1 in p53-R273H-induced cell migration.

A series of clinical and basic evidence indicates that activation of EGFR plays a critical role in NSCLC initiation and development [41, 42]. Targeting EGFR is a promising strategy for NSCLC treatment. Regimen EGFR tyrosine kinase inhibitor (TKI), such as erlotinib or gefitinib, exhibited a good benefit for NSCLC patients [42]. However, EGFR-TKI resistance is a major challenge for NSCLC treatment [43]. It has been reported that NEU1 and MMP-9 form a complex with EGFR on the cell surface that is essential for the activation of EGFR [44]. Here, we showed that NEU1 plays a role in mutant p53-mediated NSCLC cell migration. It is conceivable that NEU1 could be a potential therapeutic target for NSCLC patients harboring mutant p53 and EGFR.

\section{Acknowledgements}

We thank Professor Zhi-Xiong Xiao (Sichuan University) for helpful discussions of this manuscript. We are grateful for members of Xiao lab for stimulating discussion. We thank Dr Bert Vogelstein for kindly providing p53-R273H expression plasmid. This work was supported by National Natural Science Foundation of China (NSFC) grant (31801175) to TL, (81802951) to YY, and (81601602) to JW and China Postdoctoral Science Foundation (2020T130451 and 2018M631081) to YY.

\section{Competing Interests}

The authors have declared that no competing interest exists.

\section{References}

1. Saad MI, Rose-John S, Jenkins BJ. ADAM17: An Emerging Therapeutic Target for Lung Cancer. Cancers (Basel). 2019; 11: 1218.

2. Sun S, Schiller JH, Spinola M, Minna JD. New molecularly targeted therapies for lung cancer. J Clin Invest. 2007; 117: 2740-50.

3. Downward J. Targeting RAS signalling pathways in cancer therapy. Nat Rev Cancer. 2003; 3: 11-22.

4. Massarelli E, Varella-Garcia M, Tang X, Xavier AC, Ozburn NC, Liu DD, et al. KRAS mutation is an important predictor of resistance to therapy with epidermal growth factor receptor tyrosine kinase inhibitors in non-small-cell lung cancer. Clin Cancer Res. 2007; 13: 2890-6.

5. Vivanco I, Sawyers CL. The phosphatidylinositol 3-Kinase AKT pathway in human cancer. Nat Rev Cancer. 2002; 2: 489-501.

6. Dolgin E. The most popular genes in the human genome. Nature. 2017; 551: 427-31.

7. Hainaut P, Hernandez T, Robinson A, Rodriguez-Tome P, Flores T, Hollstein $\mathrm{M}$, et al. IARC Database of p53 gene mutations in human tumors and cell lines: updated compilation, revised formats and new visualisation tools. Nucleic Acids Res. 1998; 26: 205-13.

8. Takahashi T, Nau MM, Chiba I, Birrer MJ, Rosenberg RK, Vinocour M, et al. p53: a frequent target for genetic abnormalities in lung cancer. Science. 1989; 246: 491-4.

9. Jeong Y, Hoang NT, Lovejoy A, Stehr H, Newman AM, Gentles AJ, et al. Role of KEAP1/NRF2 and TP53 Mutations in Lung Squamous Cell Carcinoma Development and Radiation Resistance. Cancer Discov. 2017; 7: 86-101.

10. Schiller JH, Adak S, Feins RH, Keller SM, Fry WA, Livingston RB, et al. Lack of prognostic significance of p53 and K-ras mutations in primary resected non-small-cell lung cancer on E4592: a Laboratory Ancillary Study on an Eastern Cooperative Oncology Group Prospective Randomized Trial of Postoperative Adjuvant Therapy. J Clin Oncol. 2001; 19: 448-57.

11. Bergholz J, Xiao ZX. Role of p63 in Development, Tumorigenesis and Cancer Progression. Cancer Microenviron. 2012; 5: 311-22. 
12. Hafner A, Bulyk ML, Jambhekar A, Lahav G. The multiple mechanisms that regulate p53 activity and cell fate. Nat Rev Mol Cell Biol. 2019; 20: 199-210.

13. Hanel W, Marchenko N, Xu S, Yu SX, Weng W, Moll U. Two hot spot mutant p53 mouse models display differential gain of function in tumorigenesis. Cell Death Differ. 2013; 20: 898-909.

14. Goh AM, Coffill CR, Lane DP. The role of mutant p53 in human cancer. J Pathol. 2011; 223: 116-26.

15. Sabapathy K, Lane DP. Therapeutic targeting of p53: all mutants are equal, but some mutants are more equal than others. Nat Rev Clin Oncol. 2018; 15: 13-30.

16. Wang Z, Li C, Li Y, Guo X, Yan Z, Gao F, et al. DpdtbA-Induced Growth Inhibition in Human Esophageal Cancer Cells Involved Inactivation of the p53/EGFR/AKT Pathway. Oxid Med Cell Longev. 2019; 2019: 5414670.

17. Lv T, Wu X, Sun L, Hu Q, Wan Y, Wang L, et al. p53-R273H upregulates neuropilin-2 to promote cell mobility and tumor metastasis. Cell death \& disease. 2017; 8: e2995.

18. Muller PA, Caswell PT, Doyle B, Iwanicki MP, Tan EH, Karim S, et al. Mutant p53 drives invasion by promoting integrin recycling. Cell. 2009; 139: 1327-41.

19. Adorno M, Cordenonsi M, Montagner M, Dupont S, Wong C, Hann B, et al. A Mutant-p53/Smad complex opposes p63 to empower TGFbeta-induced metastasis. Cell. 2009; 137: 87-98.

20. Zhou G, Wang J, Zhao M, Xie TX, Tanaka N, Sano D, et al. Gain-of-function mutant p53 promotes cell growth and cancer cell metabolism via inhibition of AMPK activation. Mol Cell. 2014; 54: 960-74.

21. Muller PA, Trinidad AG, Timpson P, Morton JP, Zanivan S, van den Berghe PV, et al. Mutant p53 enhances MET trafficking and signalling to drive cell scattering and invasion. Oncogene. 2013; 32: 1252-65.

22. Fougerat A, Pan X, Smutova V, Heveker N, Cairo CW, Issad T, et al. Neuraminidase 1 activates insulin receptor and reverses insulin resistance in obese mice. Mol Metab. 2018; 12: 76-88.

23. Monti E, Miyagi T. Structure and Function of Mammalian Sialidases. Top Curr Chem. 2015; 366: 183-208.

24. Haxho F, Neufeld RJ, Szewczuk MR. Neuraminidase-1: a novel therapeutic target in multistage tumorigenesis. Oncotarget. 2016; 7: 40860-81.

25. Chang S, He S, Qiu G, Lu J, Wang J, Liu J, et al. MicroRNA-125b promotes invasion and metastasis of gastric cancer by targeting STARD13 and NEU1. Tumour Biol. 2016; 37: 12141-51.

26. Hyun SW, Liu A, Liu Z, Cross AS, Verceles AC, Magesh S, et al. The NEU1-selective sialidase inhibitor, C9-butyl-amide-DANA, blocks sialidase activity and NEU1-mediated bioactivities in human lung in vitro and murine lung in vivo. Glycobiology. 2016; 26: 834-49.

27. Ren LR, Zhang LP, Huang SY, Zhu YF, Li WJ, Fang SY, et al. Effects of sialidase NEU1 siRNA on proliferation, apoptosis, and invasion in human ovarian cancer. Mol Cell Biochem. 2016; 411: 213-9.

28. Wu J, Liang S, Bergholz J, He H, Walsh EM, Zhang Y, et al. DeltaNp63alpha activates CD82 metastasis suppressor to inhibit cancer cell invasion. Cell death \& disease. 2014; 5: e1280.

29. Hu L, Liang S, Chen H, Lv T, Wu J, Chen D, et al. DeltaNp63alpha is a common inhibitory target in oncogenic PI3K/Ras/Her2-induced cell motility and tumor metastasis. Proc Natl Acad Sci U S A. 2017; 114: E3964-e73.

30. Deben C, Deschoolmeester V, De Waele J, Jacobs J, Van den Bossche J, Wouters A, et al. Hypoxia-Induced Cisplatin Resistance in Non-Small Cell Lung Cancer Cells Is Mediated by HIF-1alpha and Mutant p53 and Can Be Overcome by Induction of Oxidative Stress. Cancers (Basel). 2018; 10: 126.

31. Wang W, Cheng B, Miao L, Mei Y, Wu M. Mutant p53-R273H gains new function in sustained activation of EGFR signaling via suppressing miR-27a expression. Cell death \& disease. 2013; 4: e574.

32. Wang Y, Li J, Gao Y, Luo Y, Luo H, Wang L, et al. Hippo kinases regulate cell junctions to inhibit tumor metastasis in response to oxidative stress. Redox Biol. 2019; 26: 101233.

33. Uemura T, Shiozaki K, Yamaguchi K, Miyazaki S, Satomi S, Kato K, et al. Contribution of sialidase NEU1 to suppression of metastasis of human colon cancer cells through desialylation of integrin beta4. Oncogene. 2009; 28: 1218-29.

34. Muller PA, Vousden KH. Mutant p53 in cancer: new functions and therapeutic opportunities. Cancer cell. 2014; 25: 304-17.

35. Shepherd FA, Lacas B, Le Teuff G, Hainaut P, Janne PA, Pignon JP, et al. Pooled Analysis of the Prognostic and Predictive Effects of TP53 Comutation Status Combined With KRAS or EGFR Mutation in Early-Stage Resected Non-Small-Cell Lung Cancer in Four Trials of Adjuvant Chemotherapy. J Clin Oncol. 2017; 35: 2018-27.

36. Nagarajan S, Bedi U, Budida A, Hamdan FH, Mishra VK, Najafova Z, et al. BRD4 promotes p63 and GRHL3 expression downstream of FOXO in mammary epithelial cells. Nucleic Acids Res. 2017; 45: 3130-45.

37. Quante T, Otto B, Brazdova M, Kejnovska I, Deppert W, Tolstonog GV. Mutant p53 is a transcriptional co-factor that binds to G-rich regulatory regions of active genes and generates transcriptional plasticity. Cell Cycle. 2012; 11: 3290-303.

38. Seyrantepe V, Poupetova H, Froissart R, Zabot MT, Maire I, Pshezhetsky AV. Molecular pathology of NEU1 gene in sialidosis. Hum Mutat. 2003; 22: 343-52.

39. Miyagi T, Takahashi K, Yamamoto K, Shiozaki K, Yamaguchi K. Biological and Pathological Roles of Ganglioside Sialidases. Prog Mol Biol Transl Sci. 2018; 156: 121-50.

40. An T, Zhang Z, Li Y, Yi J, Zhang W, Chen D, et al. Integrin beta1-Mediated Cell(-)Cell Adhesion Augments Metformin-Induced Anoikis. Int J Mol Sci. 2019; 20: 1161
41. Killock D. A new standard for EGFR-mutant NSCLC? Nat Rev Clin Oncol. 2019; 16: 593.

42. Arbour KC, Riely GJ. Systemic Therapy for Locally Advanced and Metastatic Non-Small Cell Lung Cancer: A Review. Jama. 2019; 322: 764-74.

43. Raoof S, Mulford IJ, Frisco-Cabanos H, Nangia V, Timonina D, Labrot E, et al. Targeting FGFR overcomes EMT-mediated resistance in EGFR mutant non-small cell lung cancer. Oncogene. 2019; 38: 6399-413.

44. Gilmour AM, Abdulkhalek S, Cheng TS, Alghamdi F, Jayanth P, O'Shea LK, et al. A novel epidermal growth factor receptor-signaling platform and its targeted translation in pancreatic cancer. Cell Signal. 2013; 25: 2587-603. 